\title{
Limits for entanglement distribution with separable states
}

\author{
Alexander Streltsov, ${ }^{\text {* }}$ Hermann Kampermann, and Dagmar Bruß \\ Heinrich-Heine-Universität Düsseldorf, Institut für Theoretische Physik III, D-40225 Düsseldorf, Germany
}

\begin{abstract}
Entanglement distribution with separable states has recently attracted considerable attention. Recent results suggest that quantum discord - a measure for quantum correlations beyond entanglement - is responsible for this counterintuitive phenomenon. In this work we study this question from a different perspective, and find minimal requirements for a separable state to be useful for entanglement distribution. Surprisingly, we find that the presence of quantum discord is not sufficient to ensure entanglement distribution: there exist states with nonzero quantum discord which nevertheless cannot be used for entanglement distribution. As a result, we show that entanglement distribution is not possible with rank two separable states. Our work sheds new light on the task of entanglement distribution with separable states, and reveals a new classification of quantum states with respect to their usefulness for this task.
\end{abstract}

A fundamental task in quantum information processing is the distribution of entanglement between two distant parties. It has been shown in [1] that, counterintuitively, this task can be achieved by sending a particle which exhibits no entanglement with the rest of the system. Very recently, such entanglement distribution with separable states has been demonstrated experimentally in physical systems with continuous [2, 3] and discrete variables [4]. Quantum discord, a novel type of quantum correlations going beyond entanglement [5- -7$]$, has been identified as the figure of merit for this puzzling phenomenon [8. 9]. This finding is in accordance with earlier results, supporting the crucial role of quantum discord and related quantifiers of quantum correlations [10-15] in quantum information theory. These results include thermodynamical approaches [16. 17], and the relations to entanglement creation in the quantum measurement process [18, 20] and to entanglement consumption in quantum state merging [21, 22]. Recently, the role of quantum discord for quantum metrology [23, 24], encoding [25] and sharing [26, 27] of information has also been subjected to scrutiny. Quantum discord was further proposed to be the figure of merit for the quantum computing protocol known as DQC1 [28] and for the task of remote state preparation [29]. While some of the arguments are still controversial [14, 30, 31], they have led to a fruitful debate about the role of general quantum correlations in quantum information theory which goes on until the present day [32, 33].

In this Letter, we aim to find minimal requirements for entanglement distribution with separable states. To this end we consider a general distribution protocol, and identify properties for a separable state to be a resource for entanglement distribution. In the following, we call a (not necessarily separable) quantum state $\rho^{A B}$ useful for entanglement distribution, if it is possible to divide the party $B$ in two parties $B_{1}$ and $B_{2}$ in such a way, that sending the particle $A$ from side 1 to side 2 leads to an increase of entanglement [34]:

$$
E^{B_{1} \mid B_{2} A}>E^{A B_{1} \mid B_{2}}
$$

see also Fig. 1] Certainly, any entangled state is useful for entanglement distribution, as can be seen by giving the full system $B$ to side 1 , i.e., $B_{1}=B$. This implies that the presence of entanglement between $A$ and $B$ is sufficient for entanglement distribution. On the other hand, the finding that entanglement

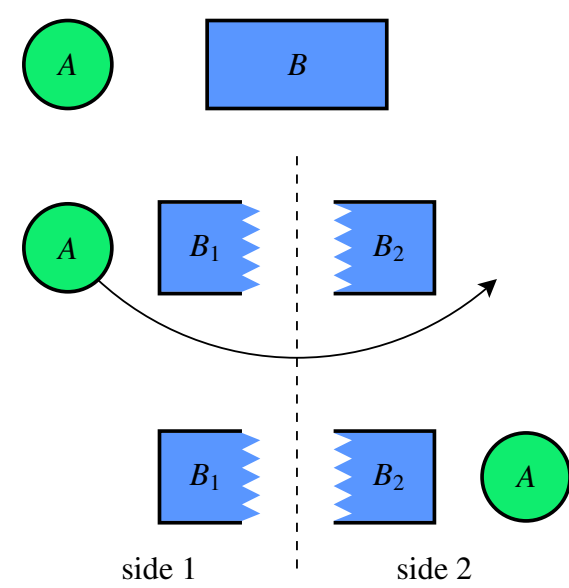

Figure 1. A quantum system consisting of two parties $A$ and $B$ (upper figure) is useful for entanglement distribution, if the party $B$ can be divided in two parties $B_{1}$ and $B_{2}$ (middle figure) in such a way, that sending the particle $A$ from side 1 to side 2 leads to an increase of entanglement (lower figure).

can be distributed with separable states [1] demonstrates that the presence of entanglement between $A$ and $B$ is not necessary, and that, in general, some other kind of quantum correlations beyond entanglement is responsible for this process.

Recently, quantum discord was identified as the key resource for entanglement distribution: the distribution of any finite amount of entanglement needs the transmission of at least the same amount of quantum discord [8, 9]. These results show that - in contrast to entanglement - quantum discord is implicitly required, if two parties wish to increase the amount of entanglement between them. As a consequence, all classical-quantum states, i.e., states of the form

$$
\rho_{\mathrm{cq}}=\sum_{i} p_{i}|i\rangle\left\langle\left. i\right|^{A} \otimes \rho_{i}^{B}\right.
$$

with $\langle i \mid j\rangle=\delta_{i j}$, cannot be used for entanglement distribution, since all those states have zero quantum discord [6]. On the other hand, the results presented in [8, 9] support the intuition that the presence of quantum discord in a quantum state $\rho^{A B}$ 
already ensures its usefulness for entanglement distribution. This idea leads us to the main question of this Letter: Are all states with nonzero quantum discord useful for entanglement distribution?

For approaching the answer to this question, we first consider the most simple class of potentially useful separable states:

$\rho^{A B}=p\left|\psi_{1}\right\rangle\left\langle\left.\psi_{1}\right|^{A} \otimes \mid \phi_{1}\right\rangle\left\langle\left.\phi_{1}\right|^{B}+(1-p) \mid \psi_{2}\right\rangle\left\langle\left.\psi_{2}\right|^{A} \otimes \mid \phi_{2}\right\rangle\left\langle\left.\phi_{2}\right|^{B}\right.$,

which is a mixture of two product states $\left|\psi_{1}^{A}\right\rangle\left|\phi_{1}^{B}\right\rangle$ and $\left|\psi_{2}^{A}\right\rangle\left|\phi_{2}^{B}\right\rangle$ with corresponding probabilities $p>0$ and $(1-p)>0$. Noting that this state has nonzero discord for a generic choice of the states $\left|\psi_{i}^{A}\right\rangle,\left|\phi_{i}^{B}\right\rangle$ and the probability $p$, it is reasonable to conjecture that this state is generically useful for entanglement distribution. Surprisingly, as we will see in the following, this intuition is not correct. This implies that the answer to the question stated above is negative, leading to strong limitations on entanglement distribution with separable states.

In the following we will show that the state given in Eq. (3) cannot be used for entanglement distribution, regardless of the choice of the states $\left|\psi_{i}^{A}\right\rangle,\left|\phi_{i}^{B}\right\rangle$ and the probability $p$. In particular, we will show that for any division of the party $B$ in two parties $B_{1}$ and $B_{2}$, sending the particle $A$ from one side to the other will never change the amount of entanglement:

$$
E_{n}^{B_{1} \mid B_{2} A}=E_{n}^{A B_{1} \mid B_{2}} .
$$

Here, $E_{n}$ is the negativity, defined for a state $\rho=\rho^{X Y}$ as $E_{n}^{X \mid Y}=\sum_{i}\left|\lambda_{i}\right|$, where $\lambda_{i}<0$ are the negative eigenvalues of the matrix $\rho^{T_{X}}$ [35, 36], and the superscript $T_{X}$ denotes partial transposition with respect to the party or parties in $X$. As the only computable quantifier of entanglement known today, the negativity is widely used in quantum information theory [37]. Since the negativity is zero only on separable and bound entangled states [38], and bound entanglement is known to be absent in bipartite states with rank smaller than four [39-41], $E_{n}$ is a faithful quantifier of entanglement for the states presented in Eq. (3).

For proving Eq. (4) we consider the partially transposed density matrices $\rho^{T_{B_{1}}}$ and $\rho^{T_{A B_{1}}}$ of the total state $\rho=\rho^{A B}=$ $\rho^{A B_{1} B_{2}}$ given in Eq. (3), where $B_{1}$ and $B_{2}$ are two subsystems of $B$. In particular, we will show that the matrices $\rho^{T_{B_{1}}}$ and $\rho^{T_{A B_{1}}}$ are equal up to a unitary, and thus share the same set of eigenvalues. For showing this, we start with the partially transposed density matrix

$$
\rho^{T_{B_{1}}}=p\left|\psi_{1}\right\rangle\left\langle\left.\psi_{1}\right|^{A} \otimes M_{1}^{B}+(1-p) \mid \psi_{2}\right\rangle\left\langle\left.\psi_{2}\right|^{A} \otimes M_{2}^{B}\right.
$$

with $M_{1}^{B}=\left(\left|\phi_{1}\right\rangle\left\langle\phi_{1}\right|\right)^{T_{B_{1}}}$ and $M_{2}^{B}=\left(\left|\phi_{2}\right\rangle\left\langle\phi_{2}\right|\right)^{T_{B_{1}}}$. In the next step we will show that a partial transposition of this matrix $\rho^{T_{B_{1}}}$ with respect to the subsystem $A$ is equivalent to a unitary, i.e., $\rho^{T_{A B_{1}}}=U \rho^{T_{B_{1}}} U^{\dagger}$. This can be seen by considering the Bloch vectors $\mathbf{r}$ and $\mathbf{s}$ corresponding to the states $\left|\psi_{1}^{A}\right\rangle$ and $\left|\psi_{2}^{A}\right\rangle$, i.e., $\left|\psi_{1}\right\rangle\left\langle\left.\psi_{1}\right|^{A}=\frac{1}{2}\left(\mathbb{1}+\sum_{i} r_{i} \sigma_{i}\right)\right.$ and $\left.\mid \psi_{2}\right\rangle\left\langle\left.\psi_{2}\right|^{A}=\frac{1}{2}(\mathbb{1}+\right.$ $\sum_{i} s_{i} \sigma_{i}$ ) with Pauli matrices $\sigma_{i}$ [42]. The transposition of the states $\left|\psi_{1}^{A}\right\rangle$ and $\left|\psi_{2}^{A}\right\rangle$ takes them to new states $\left|\tilde{\psi}_{1}^{A}\right\rangle$ and $\left|\tilde{\psi}_{2}^{A}\right\rangle$ with Bloch vectors $\tilde{\mathbf{r}}$ and $\tilde{\mathbf{s}}$. At this point, it is crucial to note that the product of the Bloch vectors does not change under transposition: $\tilde{\mathbf{r}} \cdot \tilde{\mathbf{s}}=\mathbf{r} \cdot \mathbf{s}$. This implies that the transposition of the subsystem $A$ is equivalent to a joint rotation of the Bloch vectors $\mathbf{r} \rightarrow \tilde{\mathbf{r}}$ and $\mathbf{s} \rightarrow \tilde{\mathbf{s}}$, which on the other hand corresponds to a unitary acting on the subsystem $A$. This proves that the matrices $\rho^{T_{B_{1}}}$ and $\rho^{T_{A B_{1}}}$ are equal up to a unitary, implying that the eigenvalues of both matrices must be the same. Starting from this result, Eq. (4) is seen to be correct by recalling that the negativity $E_{n}^{X \mid Y}$ depends only on the eigenvalues of the partially transposed density matrix $\rho^{T_{X}}$.

The results presented so far imply crucial constraints on the possibility to distribute entanglement with separable states. In particular we have seen that the distribution of entanglement is not possible, if the corresponding separable state is a mixture of two pure product states, see Eq. (3). In the next step we will see that this limitation can be surpassed, if the pure states $\left|\psi_{i}^{A}\right\rangle$ in Eq. 3 are replaced by mixed states $\rho_{i}^{A}$. In this case the total state takes the form

$$
\rho^{A B}=p \cdot \rho_{1}^{A} \otimes\left|\phi_{1}\right\rangle\left\langle\left.\phi_{1}\right|^{B}+(1-p) \cdot \rho_{2}^{A} \otimes \mid \phi_{2}\right\rangle\left\langle\left.\phi_{2}\right|^{B} .\right.
$$

The use of this state for entanglement distribution can be demonstrated by a proper choice of the states $\rho_{i}^{A}$ and $\left|\phi_{i}^{B}\right\rangle$. This can be achieved by defining the states $\rho_{i}^{A}$ of the subsystem $A$ as follows:

$$
\begin{aligned}
\rho_{1}^{A} & =\frac{1}{4}|0\rangle\left\langle 0\left|+\frac{3}{4}\right| 1\right\rangle\langle 1|, \\
\rho_{2}^{A} & =\frac{1}{2}|a\rangle\left\langle a\left|+\frac{1}{2}\right| b\right\rangle\langle b|,
\end{aligned}
$$

where $|a\rangle$ and $|b\rangle$ are nonorthogonal qutrit states, defined as $|a\rangle=(|0\rangle+|1\rangle+|2\rangle) / \sqrt{3}$ and $|b\rangle=(|0\rangle+i|1\rangle) / \sqrt{2}$. Finally, the party $B$ consists of two subsystems $B_{1}$ and $B_{2}$, and the corresponding states $\left|\phi_{i}^{B}\right\rangle$ can be chosen as $\left|\phi_{1}^{B}\right\rangle=(|00\rangle+|01\rangle+$ $i|11\rangle) / \sqrt{3}$ and $\left|\phi_{2}^{B}\right\rangle=(\sqrt{8}|00\rangle+|11\rangle) / 3$. As can be seen from

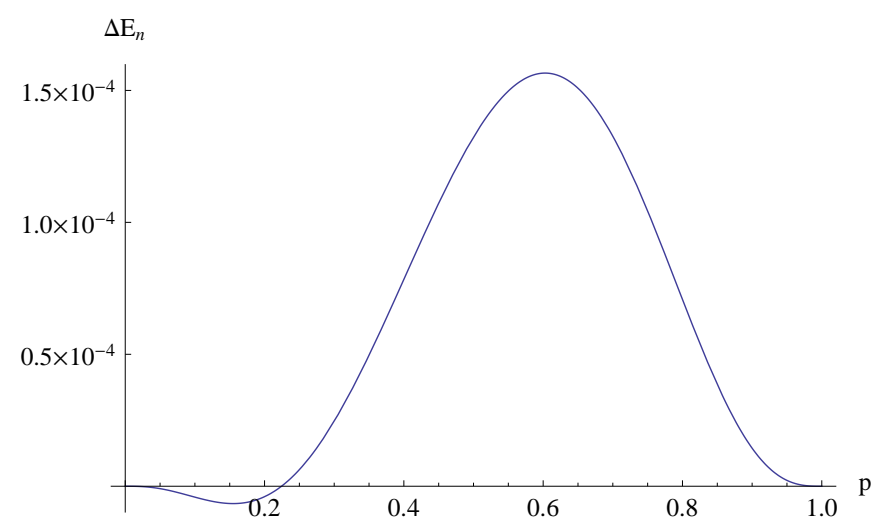

Figure 2. Entanglement distribution with separable states: the plot shows the amount of distributed entanglement $\Delta E_{n}=E_{n}^{B_{1} \mid B_{2} A}-$ $E_{n}^{A B_{1} \mid B_{2}}$ for the state given in Eq. (6) as function of the probability $p$. The state allows to distribute a finite amount of entanglement $\Delta E_{n}>0$ in the range $p^{\prime}<p<1$ with $p^{\prime} \approx 0.22$. 
the difference $\Delta E_{n}=E_{n}^{B_{1} \mid B_{2} A}-E_{n}^{A B_{1} \mid B_{2}}$, shown in Fig. 2 as a function of $p$, this particular setting allows to distribute a finite amount of entanglement $\Delta E_{n}>0$ in the range $p^{\prime}<p<1$ for $p^{\prime} \approx 0.22$.

The example presented above should be regarded as a proof of principle: it explicitly demonstrates that some separable states which are mixtures of only two product states can in principle - be used for entanglement distribution. In particular, we have seen that a successful distribution of entanglement can be achieved by a specific choice of mixed qutrit states $\rho_{1}^{A}$ and $\rho_{2}^{A}$, see Eq. 77. As we will see in the following, it is indeed crucial that the transmitted particle $A$ is not a qubit: for entanglement distribution with separable states as given in Eq. (6) the dimension of $A$ needs to be at least three. For proving this statement it is enough to show that for a twodimensional subsystem $A$ the state given in Eq. (6) cannot be used for entanglement distribution, i.e., Eq. (4) is satisfied. This can be seen by observing that the arguments given in the proof of Eq. (4) for mixtures of two pure product states remain valid if the pure states $\left|\psi_{1}^{A}\right\rangle$ and $\left|\psi_{2}^{A}\right\rangle$ are replaced by arbitrary qubit states $\rho_{1}^{A}$ and $\rho_{2}^{A}$.

On the one hand, we have seen that entanglement distribution with separable states is impossible, if the separable state is a mixture of two pure product states only. On the other hand, we have also demonstrated a possibility to avoid this problem by using two mixed states $\rho_{1}^{A}$ and $\rho_{2}^{A}$ for the exchanged particle $A$. In the next step we will show that mixedness of both states is essential: entanglement distribution is not possible if $\rho_{1}^{A}$ or $\rho_{2}^{A}$ is pure, regardless of the dimension of the exchanged particle $A$. We will prove this statement by showing that Eq. (4) is satisfied for all states given in Eq. (6) as long as either $\rho_{1}^{A}$ or $\rho_{2}^{A}$ is pure. Without loss of generality we can assume that $\rho_{1}^{A}=|\psi\rangle\left\langle\left.\psi\right|^{A}\right.$ is pure, and the state $\rho_{2}^{A}=\tau^{A}$ is diagonal in the computational basis: $\tau^{A}=\sum_{i} \lambda_{i}|i\rangle\left\langle\left. i\right|^{A}\right.$ [43]. Using similar lines of reasoning as above we will prove the validity of Eq. (4) by showing that the partially transposed density matrices $\rho^{T_{B_{1}}}$ and $\rho^{T_{A B_{1}}}$ are equal up to a unitary. In particular, the matrix $\rho^{T_{B_{1}}}$ has now the form

$$
\rho^{T_{B_{1}}}=p|\psi\rangle\left\langle\left.\psi\right|^{A} \otimes M_{1}^{B}+(1-p) \cdot \tau^{A} \otimes M_{2}^{B},\right.
$$

where $M_{1}^{B}=\left(\left|\phi_{1}\right\rangle\left\langle\phi_{1}\right|\right)^{T_{B_{1}}}$ and $M_{2}^{B}=\left(\left|\phi_{2}\right\rangle\left\langle\phi_{2}\right|\right)^{T_{B_{1}}}$. The matrix $\rho^{T_{A B_{1}}}$ can be obtained from this expression by performing partial transposition on the subsystem $A$ :

$$
\rho^{T_{A B_{1}}}=p|\tilde{\psi}\rangle\left\langle\left.\tilde{\psi}\right|^{A} \otimes M_{1}^{B}+(1-p) \cdot \tau^{A} \otimes M_{2}^{B} .\right.
$$

Here, we used the fact that $\tau^{A}=\sum_{i} \lambda_{i}|i\rangle\left\langle\left. i\right|^{A}\right.$ is diagonal in the computational basis, and thus does not change under transposition. The relation between the state $\left|\psi^{A}\right\rangle$ and the transposed state $\left|\tilde{\psi}^{A}\right\rangle$ can be seen by expanding both states in the computational basis: $\left|\tilde{\psi}^{A}\right\rangle=\sum_{j} c_{j}^{*}\left|j^{A}\right\rangle$, where $c_{j}$ are the coefficients of the state $\left|\psi^{A}\right\rangle$, i.e., $\left|\psi^{A}\right\rangle=\sum_{j} c_{j}\left|j^{A}\right\rangle$. In the final step it is important to note that the states $\left|\psi^{A}\right\rangle$ and $\left|\tilde{\psi}^{A}\right\rangle=U\left|\psi^{A}\right\rangle$ are related by the unitary $U=\sum_{j} e^{-2 i \phi_{j}}|j\rangle\left\langle\left. j\right|^{A}\right.$, where $\phi_{j}$ is the phase corresponding to the coefficient $c_{j}=\left|c_{j}\right| \cdot e^{i \phi_{j}}$. Since this unitary is diagonal in the computational basis, it does not change the state $\tau^{A}$, and thus we obtain the desired result: $\rho^{T_{A B_{1}}}=U \rho^{T_{B_{1}}} U^{\dagger}$. This proves that for a successful distribution of entanglement with separable states as given in Eq. (6) both states $\rho_{1}^{A}$ and $\rho_{2}^{A}$ must be mixed.

The results presented above indicate that the structure of the separable state - and in particular the number of product states in its mixture - is crucial, if the separable state is to be used as a resource for entanglement distribution. While a mixture of only two product states does not allow to distribute any entanglement by sending a single qubit, this limitation disappears if the exchanged particle has dimension three. We also note that this result remains valid if the pure states $\left|\phi_{i}^{B}\right\rangle$ of the subsystem $B$ are replaced by mixed states $\rho_{i}^{B}$. In particular, separable states of the form $\rho^{A B}=p \cdot \rho_{1}^{A} \otimes \rho_{1}^{B}+(1-p) \cdot \rho_{2}^{A} \otimes \rho_{2}^{B}$ can only be used for entanglement distribution if the transmitted particle $A$ has at least dimension three, and if both states $\rho_{1}^{A}$ and $\rho_{2}^{A}$ are not pure.

In the next step it is natural to ask about the situation where the separable state used for entanglement distribution is more general. As we will see in the following, qubits can still be used to distribute entanglement if the separable state is a mixture of at least three product states. This can be demonstrated on the following separable state:

$$
\rho^{A B}=\frac{1}{3} \sum_{i=1}^{3}\left|\psi_{i}\right\rangle\left\langle\left.\psi_{i}\right|^{A} \otimes \mid \phi_{i}\right\rangle\left\langle\left.\phi_{i}\right|^{B},\right.
$$

where the qubit states $\left|\psi_{i}^{A}\right\rangle$ of the transmitted particle $A$ are chosen as follows: $\left|\psi_{1}^{A}\right\rangle=(|0\rangle+|1\rangle) / \sqrt{2},\left|\psi_{2}^{A}\right\rangle=(|0\rangle+$ $i|1\rangle) / \sqrt{2}$, and $\left|\psi_{3}^{A}\right\rangle=|0\rangle$. The party $B$ consists of two subsystems $B_{1}$ and $B_{2}$, and the corresponding states $\left|\phi_{i}^{B}\right\rangle$ are defined as $\left|\phi_{1}^{B}\right\rangle=|01\rangle,\left|\phi_{2}^{B}\right\rangle=(|00\rangle+i|11\rangle) / \sqrt{2}$, and $\left|\phi_{3}^{B}\right\rangle=\cos \alpha|00\rangle+\sin \alpha|11\rangle$. As can be seen from Fig. 3. this state allows to distribute a finite amount of entanglement

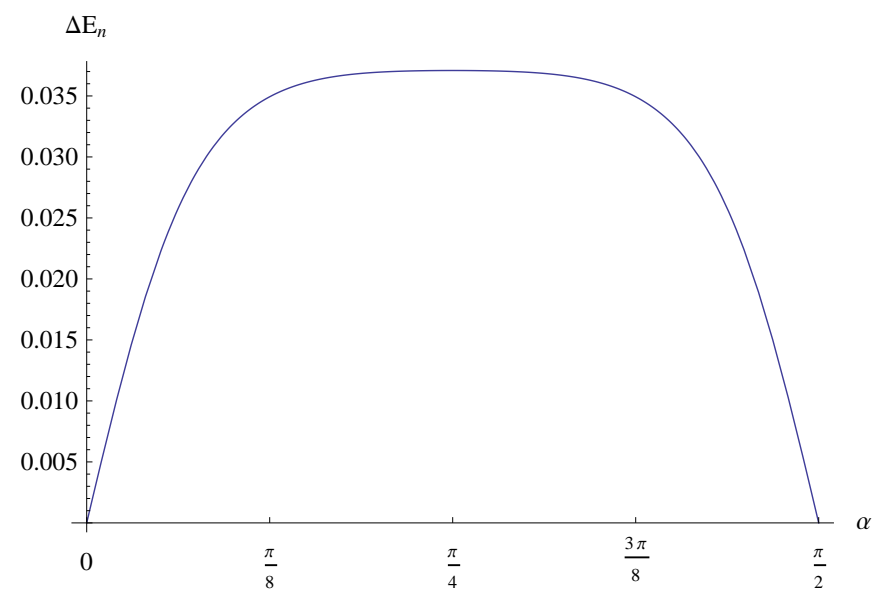

Figure 3. Entanglement distribution with separable states by sending a single qubit: the plot shows the amount of distributed entanglement $\Delta E_{n}=E_{n}^{B_{1} \mid B_{2} A}-E_{n}^{A B_{1} \mid B_{2}}$ for the state given in Eq. $[10)$ as function of the parameter $\alpha$. A finite amount of entanglement $\Delta E_{n}>0$ can be distributed in the range $0<\alpha<\pi / 2$. 
$\Delta E_{n}=E_{n}^{B_{1} \mid B_{2} A}-E_{n}^{A B_{1} \mid B_{2}}$ in the range $0<\alpha<\pi / 2$, where $\alpha$ is the parameter of the state $\left|\phi_{3}^{B}\right\rangle$.

As will become clear in a moment, the reason why the state in Eq. (10) is useful for entanglement distribution lies in the structure of the states $\left|\psi_{i}^{A}\right\rangle$. In particular, their Bloch vectors are given by $\mathbf{r}_{1}=(1,0,0)^{T}, \mathbf{r}_{2}=(0,1,0)^{T}$, and $\mathbf{r}_{3}=(0,0,1)^{T}$. Observe that these three vectors are linearly independent, i.e., they are not all in the same plane. We will see in the following that this feature is crucial for entanglement distribution, where at the same time we will generalize our results to arbitrary separable states, i.e., states of the form

$$
\rho^{A B}=\sum_{i} p_{i} \cdot \rho_{i}^{A} \otimes \rho_{i}^{B}
$$

where the exchanged particle $A$ is a qubit. Following the same arguments as in the preceding discussion we consider the partially transposed matrices

$$
\begin{aligned}
\rho^{T_{B_{1}}} & =\sum_{i} p_{i} \cdot \rho_{i}^{A} \otimes M_{i}^{B}, \\
\rho^{T_{A B_{1}}} & =\sum_{i} p_{i} \cdot \tilde{\rho}_{i}^{A} \otimes M_{i}^{B}
\end{aligned}
$$

with $M_{i}^{B}=\left(\rho_{i}^{B}\right)^{T_{B_{1}}}$. Recall that the state $\rho^{A B}$ cannot be used for entanglement distribution if the two matrices $\rho^{T_{B_{1}}}$ and $\rho^{T_{A B_{1}}}$ are equal up to a unitary. On the one hand, this is the case whenever the transposition on the subsystem $A$ of the matrix $\rho^{T_{B_{1}}}$ corresponds to a joint rotation of the Bloch vectors $\mathbf{r}_{i} \rightarrow \tilde{\mathbf{r}}_{i}$, i.e., whenever there exists a special orthogonal $3 \times 3$ matrix $O$ such that $\tilde{\mathbf{r}}_{i}=O \cdot \mathbf{r}_{i}$ [44]. Here, $\mathbf{r}_{i}$ and $\tilde{\mathbf{r}}_{i}$ are the Bloch vectors of $\rho_{i}^{A}$ and the transposed state $\tilde{\rho}_{i}^{A}$, respectively. On the other hand, it is crucial to note that the Bloch vector $\tilde{\mathbf{r}}$ corresponding to a transposed state $\tilde{\rho}=\rho^{T}$ is related to the Bloch vector $\mathbf{r}$ of the initial state $\rho$ via a reflection on the xzplane, i.e., $\left(\tilde{r}_{1}, \tilde{r}_{2}, \tilde{r}_{3}\right)=\left(r_{1},-r_{2}, r_{3}\right)$. Combining these results we can say that $\rho^{A B}$ cannot be used for entanglement distribution if for all the Bloch vectors $\mathbf{r}_{i}$ a reflection on the xz-plane is equivalent to a rotation. Note that this is always fulfilled if the number of Bloch vectors is two, in accordance with the finding that mixtures of two product states cannot be used for entanglement distribution by sending a qubit. For more than two vectors a reflection does not necessarily correspond to a rotation, supporting the finding that qubits can be used for entanglement distribution if the number of product states is more than two. Finally, we point out that reflection is equivalent to rotation for any number of Bloch vectors, whenever all the Bloch vectors are in the same plane, i.e., whenever any Bloch vector $\mathbf{r}_{i}$ can be written as a superposition of $\mathbf{r}_{1}$ and $\mathbf{r}_{2}$. This immediately leads to a generalization of our previous results: entanglement distribution with separable states by sending a single qubit is only possible if the corresponding Bloch vectors $\mathbf{r}_{i}$ are not all in the same plane.

In conclusion, we established minimal requirements for a separable state to be a resource for entanglement distribution. Here, both the dimension of the exchanged particle and the number of product terms in the decomposition play a crucial

\begin{tabular}{ccc}
\hline \hline$d_{A}$ & $n$ & Entanglement distribution possible? \\
\hline 2 & 2 & no \\
2 & 3 & yes \\
3 & 2 & yes \\
\hline \hline
\end{tabular}

Table I. Requirements on a separable state $\rho^{A B}=\sum_{i=1}^{n} p_{i} \cdot \rho_{i}^{A} \otimes \rho_{i}^{B}$ to be useful for entanglement distribution. Here, $d_{A}$ is the dimension of the exchanged particle $A$, and $n$ is the minimal number of product terms in the decomposition.

role - for a summary see Table I. These requirements were deduced from general symmetry arguments, relating the partial transpose to a rotation. Our results provide an answer to the main question of this Letter: there are states with nonzero quantum discord which cannot be used as a resource for entanglement distribution. In particular, we have shown that a separable state cannot be used for this task, if it is a mixture of only two pure product states. Since all rank two separable states are mixtures of two pure product states [45], we can conclude that entanglement distribution with separable states requires states with rank at least three. Finally, our results suggest a new classification of quantum states according to their usefulness for entanglement distribution. While all entangled states are evidently useful for this task, a separable state can only be useful if it exhibits nonzero quantum discord, and fulfills the additional requirements provided in Table $[$ The induced substructure within the set of separable states is reminiscent of the structure within the set of entangled states, which arises from being useful for distillation (free entanglement) or not being useful for distillation (bound entanglement). Due to the fact that entanglement distribution is a fundamental task in quantum information theory, the results presented in this work may be helpful to devise new protocols for entanglement distribution, and to gain new insights into the properties of quantum entanglement and general quantum correlations.

Acknowledgements: We acknowledge financial support by the German Federal Ministry of Education and Research (BMBF, project QuOReP), and ELES.

* streltsov@thphy.uni-duesseldorf.de

[1] T. S. Cubitt, F. Verstraete, W. Dür, and J. I. Cirac, Phys. Rev. Lett. 91, 037902 (2003)

[2] C. E. Vollmer, D. Schulze, T. Eberle, V. Händchen, J. Fiurasek, and R. Schnabel, arXiv:1303.1082v2

[3] C. Peuntinger, V. Chille, L. Mišta, Jr., N. Korolkova, M. Förtsch, J. Korger, C. Marquardt, and G. Leuchs, arXiv:1304.0504v1

[4] A. Fedrizzi, M. Zuppardo, G. G. Gillett, M. A. Broome, M. de Almeida, M. Paternostro, A. G. White, and T. Paterek, arXiv:1303.4634v1

[5] W. H. Zurek, Ann. Phys. (Berlin) 9, 855 (2000)

[6] H. Ollivier and W. H. Zurek, Phys. Rev. Lett. 88, 017901 (2001)

[7] L. Henderson and V. Vedral, J. Phys. A 34, 6899 (2001) 
[8] A. Streltsov, H. Kampermann, and D. Bruß, Phys. Rev. Lett. 108, 250501 (2012)

[9] T. K. Chuan, J. Maillard, K. Modi, T. Paterek, M. Paternostro, and M. Piani, Phys. Rev. Lett. 109, 070501 (2012)

[10] S. Luo, Phys. Rev. A 77, 022301 (2008)

[11] K. Modi, T. Paterek, W. Son, V. Vedral, and M. Williamson, Phys. Rev. Lett. 104, 080501 (2010).

[12] P. Giorda and M. G. A. Paris, Phys. Rev. Lett. 105, 020503 (2010)

[13] G. Adesso and A. Datta, Phys. Rev. Lett. 105, 030501 (2010)

[14] B. Dakić, V. Vedral, and C. Brukner, Phys. Rev. Lett. 105, 190502 (2010)

[15] G. L. Giorgi, B. Bellomo, F. Galve, and R. Zambrini, Phys. Rev. Lett. 107, 190501 (2011).

[16] J. Oppenheim, M. Horodecki, P. Horodecki, and R. Horodecki, Phys. Rev. Lett. 89, 180402 (2002)

[17] W. H. Zurek, Phys. Rev. A 67, 012320 (2003)

[18] A. Streltsov, H. Kampermann, and D. Bruß, Phys. Rev. Lett. 106, 160401 (2011)

[19] M. Piani, S. Gharibian, G. Adesso, J. Calsamiglia, P. Horodecki, and A. Winter, Phys. Rev. Lett. 106, 220403 (2011)

[20] G. Adesso, V. D'Ambrosio, E. Nagali, M. Piani, and F. Sciarrino, arXiv:1308.1680v1

[21] V. Madhok and A. Datta, Phys. Rev. A 83, 032323 (2011)

[22] D. Cavalcanti, L. Aolita, S. Boixo, K. Modi, M. Piani, and A. Winter, Phys. Rev. A 83, 032324 (2011)

[23] K. Modi, H. Cable, M. Williamson, and V. Vedral, Phys. Rev. X 1, 021022 (2011)

[24] D. Girolami, T. Tufarelli, and G. Adesso, Phys. Rev. Lett. 110, 240402 (2013)

[25] M. Gu, H. M. Chrzanowski, S. M. Assad, T. Symul, K. Modi, T. C. Ralph, V. Vedral, and P. K. Lam, Nat. Phys. 8, 671 (2012)

[26] M. Zwolak and W. H. Zurek, Sci. Rep. 3, 1729 (2013).

[27] A. Streltsov and W. H. Zurek, Phys. Rev. Lett. 111, 040401 (2013)

[28] A. Datta, A. Shaji, and C. M. Caves, Phys. Rev. Lett. 100, $050502(2008)$

[29] B. Dakić, Y. O. Lipp, X. Ma, M. Ringbauer, S. Kropatschek, S. Barz, T. Paterek, V. Vedral, A. Zeilinger, Č. Brukner, and
P. Walther, Nat. Phys. 8, 666 (2012)

[30] G. L. Giorgi, Phys. Rev. A 88, 022315 (2013)

[31] P. Horodecki, J. Tuziemski, P. Mazurek, and R. Horodecki, arXiv:1306.4938v1

[32] Z. Merali, Nature 474, 24 (2011).

[33] K. Modi, A. Brodutch, H. Cable, T. Paterek, and V. Vedral, Rev. Mod. Phys. 84, 1655 (2012)

[34] Here, "dividing" $B$ into $B_{1}$ and $B_{2}$ means to define a tensor product structure for the corresponding Hilbert spaces: $\mathcal{H}_{B}=$ $\mathcal{H}_{B_{1}} \otimes \mathcal{H}_{B_{2}}$.

[35] K. Życzkowski, P. Horodecki, A. Sanpera, and M. Lewenstein, Phys. Rev. A 58, 883 (1998)

[36] G. Vidal and R. F. Werner, Phys. Rev. A 65, 032314 (2002)

[37] R. Horodecki, P. Horodecki, M. Horodecki, and K. Horodecki, Rev. Mod. Phys. 81, 865 (2009)

[38] M. Horodecki, P. Horodecki, and R. Horodecki, Phys. Rev. Lett. 80, 5239 (1998)

[39] P. Horodecki, M. Lewenstein, G. Vidal, and I. Cirac, Phys. Rev. A 62, 032310 (2000)

[40] P. Horodecki, J. A. Smolin, B. M. Terhal, and A. V. Thapliyal, Theoretical Computer Science 292, 589 (2003)

[41] L. Chen and Y.-X. Chen, Phys. Rev. A 78, 022318 (2008)

[42] Since $\left|\psi_{1}^{A}\right\rangle$ and $\left|\psi_{2}^{A}\right\rangle$ span a two-dimensional subspace, this argument is not limited to qubits, but applies regardless of the dimension of the party $A$.

[43] If it was possible to violate Eq. (4) by choosing $\tau^{A}$ which is not diagonal in the computational basis, the invariance of negativity under local unitaries implies that Eq. (4) is also violated for the diagonal state $\tau_{\text {diag }}^{A}=U \tau^{A} U^{\dagger}$.

[44] Note that this argument applies only if the exchanged particle $A$ is a qubit, since in this case any rotation on the Bloch sphere corresponds to a unitary on the corresponding Hilbert space. The fact that this is no longer true for higher-dimensional systems is responsible for the effect presented in Eq. (6) and Fig. 2. it allows to distribute entanglement with mixtures of only two product states, if the exchanged particle is a qutrit.

[45] A. Sanpera, R. Tarrach, and G. Vidal, Phys. Rev. A 58, 826 (1998) 